\title{
Duhuo Jisheng decoction treatment inhibits the sodium nitroprussiate-induced apoptosis of chondrocytes through the mitochondrial-dependent signaling pathway
}

\author{
FAYUAN LIU ${ }^{1 *}$, GUOZHONG LIU $^{2 *}$, WENNA LIANG ${ }^{3}$, HONGZHI YE $^{1}$, XIAPING WENG ${ }^{1}$, \\ PINGDONG LIN ${ }^{1}$, HUITING LI ${ }^{4}$, JIASHOU CHEN ${ }^{4}$, XIANXIANG LIU ${ }^{1}$ and XIHAI LI ${ }^{1}$ \\ ${ }^{1}$ Academy of Integrative Medicine, Fujian University of Traditional Chinese Medicine, Fuzhou 350122; \\ ${ }^{2}$ The First Affiliated Hospital of Fujian Medical University, Fujian 350002; \\ ${ }^{3}$ Research Base of Traditional Chinese Medicine Syndrome, ${ }^{4}$ Fujian Key Laboratory of Integrative Medicine on Geriatrics, \\ Fujian University of Traditional Chinese Medicine, Fuzhou 350122, P.R. China
}

Received April 4, 2014; Accepted September 29, 2014

DOI: $10.3892 / \mathrm{ijmm} .2014 .1962$

\begin{abstract}
Chondrocyte apoptosis activated by the mitochondrial-dependent signaling pathway plays a crucial role in the cartilage degeneration of osteoarthritis. Duhuo Jisheng decoction (DHJSD), a herbal formula from traditional Chinese medicine, has been widely used for treating osteoarthritis (OA). However, the molecular mechanisms behind the therapeutic effect of DHJSD remain to be elucidated. In the present study, the effects of DHJSD on the mitochondrial-dependent signaling pathway in sodium nitroprussiate (SNP)-induced chondrocyte apoptosis were investigated. Chondrocytes, from the knee articular cartilage of Sprague Dawley rats, were identified by type II collagen immunohistochemistry. The chondrocytes, stimulated with or without SNP to induce apoptosis, were treated by DHJSD for various concentrations and times. The viability of SNP-induced chondrocytes treated with DHJSD was enhanced compared to SNP-induced chondrocytes in a dose- and time-dependent manner, as assessed by the MTT assay. The apoptosis of SNP-induced chondrocytes treated by DHJSD was significantly decreased compared to SNP-induced chondrocyte, as shown by 4',6-diamidino-2-phenylindole and Annexin V/propidium iodide staining. The mitochondrial membrane potential $(\Delta \Psi \mathrm{m})$ of SNP-induced chondrocytes treated by DHJSD was significantly decreased compared to SNP-induced chondrocyte, as shown by JC-1 staining. To understand the mechanism, the mRNA and protein levels of
\end{abstract}

Correspondence to: Dr Xihai Li, Academy of Integrative Medicine, Fujian University of Traditional Chinese Medicine, 1 Huatuo, University Town, Shangjie Minhou, Fuzhou 350122, P.R. China E-mail: lixihai79dahai@163.com

*Contributed equally

Key words: osteoarthritis, Duhuo Jisheng decoction, chondrocyte, apoptosis
Bax, B-cell lymphoma 2 (Bcl-2), caspase-9 and caspase-3 were detected by reverse transcription-polymerase chain reaction and western blot analysis, respectively. In SNP-induced chondrocyte treated by DHJSD, the Bcl-2 expression was increased, whereas the expression of Bax, caspase-9 and caspase-3 was decreased compared to SNP-induced chondrocyte. Taken together, these results indicated that DHJSD inhibits the apoptosis of SNP-induced chondrocyte by the mitochondrial-dependent apoptotic pathway, and this may partly explain its therapeutic efficacy for OA.

\section{Introduction}

Osteoarthritis (OA), one of the most frequent degenerative joint diseases, is characterized by the progressive degeneration of articular cartilage and leads to limitation of joint movement, joint deformity, tenderness, inflammation and severe pain. Cartilage aging and chondrocyte senescence are caused by the apoptosis of chondrocytes that have been hypothesized to be a crucial event in the development of OA (1). Chondrocytes, the only cell type resident in articular cartilage, have a limited ability for proliferation and self-repair. Therefore, reducing chondrocyte apoptosis may be a potential way for OA treatment.

Apoptosis is activated by two major pathways; the extrinsic (death signals are mediated through cell surface receptors) and intrinsic pathways (death signals are integrated at the mitochondrial level, which is also referred to as the mitochondrion-dependent apoptotic signaling pathway). The pathways eventually lead to the activation of nucleases and caspases, resulting in cell death $(2,3)$. The mitochondrial-dependent signaling pathway includes loss of mitochondrial membrane potential $(\Delta \Psi \mathrm{m})$, regulation of the B-cell lymphoma 2 (Bcl-2) family of proteins that are the main regulators of this deadly switch, and activation of caspases, such as caspase-9 and caspase-3 $(4,5)$. Mitochondria are associated with the apoptosis of chondrocytes in OA (6). Therefore, decreasing apoptosis through regulating the effect of the $\mathrm{Bcl}-2$ family proteins and caspases on the mitochondrial-dependent signaling pathway 
has been the main focus in relieving the progressive degeneration of articular cartilage.

Currently, the treatments for OA are short-term or ineffective, and these often have undesirable side-effects that make treatment unsustainable $(7,8)$. Natural products, such as traditional Chinese medicine (TCM), have certain evidence for improving the efficacy for OA through decreasing joint pain and dysfunction, and preventing and delaying the cartilage degeneration $(9,10)$. According to the TCM theory, OA belonging to the category of $B I$-syndrome is mainly considered to include liver and kidney deficiency, retention of cold-damp, phlegm and blood stagnation in the knees. Duhuo Jisheng decoction (DHJSD), a herbal formula from TCM, has been proved effective in OA treatment by relieving pain, reducing joint stiffness and improving mobility and quality of life (11). A previous study has proven that DHJSD has potential cooperation and polypharmacology against OA (12). However, the mechanisms of DHJSD on the apoptosis of chondrocyte remain to be elucidated. To extend the clinical treatment of OA with a novel approach for TCM therapy and to aid in establishing a scientific foundation for further research, the present study was based on the SNP-induced chondrocyte apoptosis model to determine whether DHJSD inhibits the apoptosis of chondrocytes by the mitochondrial-dependent signaling pathway, thus delaying degeneration of articular cartilage.

\section{Materials and methods}

DHJSD aqueous extract preparation. DHJSD consists of 15 plant species as follows: 9 g of Radix Angelicae pubescentis, $6 \mathrm{~g}$ each of Ramulus Loranthi, Radix Gentianae Macrophyllae, Radix Saposhnikoviae, Herba Asari, Rhizoma Chuanxiong, Radix Angelicae Sinensis, Radix Rehmanniae, Radix Paeoniae Alba, Cortex Cinnamomi, Poria Cocos, Ulmoidis Cortex Eucommiae, Radix Achyranthis Bidentatae, Panax ginseng and Radix Glycyrrhizae. The components were mixed and extracted with standard methods according to the Chinese Pharmacopoeia (13). These were soaked in distilled water, boiled for $30 \mathrm{~min}$ twice and the solution was filtered and concentrated. The filtrate of DHJSD was dissolved in Dulbecco's modified Eagle's medium containing 10\% fetal bovine serum at a concentration of $10 \mathrm{mg} / \mathrm{ml}$, and subsequently filtered through a filter and stored at $4^{\circ} \mathrm{C}$.

Isolation, identification and treatment of chondrocytes. Chondrocytes were isolated from knee cartilage of 4-week-old male Sprague Dawley rats (Super-BK Laboratory Animal, Co. Shanghai, China), cultured and identified as described previously $(14,15)$. The present study was reviewed and approved by the Ethics Committee of Science and Technology of China, and all the animals complied with the guidance suggestions for the care and use of laboratory animals at Fujian University of TCM (Fuzhou, China). Chondrocytes were treated with or without $1 \mathrm{mM}$ SNP (Sigma, Upper Saddle River, NJ, USA), as described previously (14), and various concentrations of DHJSD. The images of chondrocytic morphology were captured by phase-contrast (Olympus, Tokyo, Japan) and scanning electron microscopes (SEM; Philips XL30; Hitachi, Tokyo, Japan). Apoptosis was detected using 4',6-diamidino-2-phenylindole (DAPI) staining followed by a fluorescent microscope
(Olympus), Annexin V/propidium iodide (PI) staining and JC-staining followed by fluorescence-activated cell sorting (FACS) using the FACS caliber (Becton-Dickinson, CA, USA). The mRNA and protein expressions of Bcl-2, Bax, caspase- 9 and caspase- 3 by RT-PCR and western blot analysis, respectively.

MTT assay. Chondrocytes were seeded in a 96-well plates (100 $\mu \mathrm{l} /$ well) at a density of $5 \times 10^{4}$ cells $/ \mathrm{ml}$ and cultured for $24 \mathrm{~h}$. They were subsequently treated with or without $1 \mathrm{mM}$ SNP and various concentrations of DHJSD (200, 300, 400, 500 and $600 \mu \mathrm{g} / \mathrm{ml}$ ) for $12,24,36,48$ or $72 \mathrm{~h}$, respectively. After treatment, the medium was changed by $100 \mu 11 \mathrm{mg} / \mathrm{ml}$ MTT (Sigma) at $37^{\circ} \mathrm{C}$ for $4 \mathrm{~h}$, and replaced with $150 \mu \mathrm{l}$ dimethyl sulfoxide and agitated for $10 \mathrm{~min}$. The cells were analyzed on an ELISA reader (Model EXL 800; BioTek Instruments, Inc., Winooski, VT, USA) using a 490-nm wave length.

SEM observation. After treatment, the chondrocytes underwent post-fixation with $1 \%$ osmium in $0.1 \mathrm{M} \mathrm{Na-cacodylate}$ and dehydrated in ethanol solutions and lyophilized. They were subsequently coated with gold in a sputtering device. Images were captured under a Philips XL30 SEM.

Assessment of chondrocyte apoptosis by DAPI staining. The adherent cells were washed three times with ice-cold phosphate-buffered saline (PBS), fixed with $4 \%$ neutral formaldehyde for $15 \mathrm{~min}$ and washed three times with ice-cold PBS. The cells were subsequently incubated in $5 \mu \mathrm{g} / \mathrm{ml}$ DAPI for $5 \mathrm{~min}$, washed three times and observed under a fluorescent microscope.

Detection of apoptosis by flow cytometry analysis with Annexin V/PI staining and JC-1 staining. The apoptosis rate of the chondrocytes was measured using an Annexin V/PI kit (KeyGEN BioTECH, Nanjing, China) by FACS. To evaluate for the loss of $\Delta \Psi \mathrm{m}$, the collected cells were incubated with the JC-1 kit (KeyGEN BioTECH) and the processed cells were analyzed by FACS. All the staining was performed according to the manufacturer's instructions (14).

RNA extraction and reverse transcription-polymerase chain reaction $(R T-P C R)$ analysis. Total RNA was extracted from the treated cells according to the standard instructions using the TRIzol reagent (Invitrogen, Grand Island, NY, USA). RNA $(1 \mu \mathrm{g})$ was reverse transcribed into cDNA according to the instructions provided by the manufacturer. The cDNA was detected for the mRNA expression of Bax, Bcl-2, caspase-3, caspase- 9 and $\beta$-actin by RT-PCR. Primer sequences were as follows: Bax forward, 5'-GGC GAT GAA CTG GAC AAC-3'; and reverse, 5'-TCC CGA AGT AGG AAA GGA g-3'; $\mathrm{Bcl}$-2 forward, 5'-CCC TGG CAT CTT CTC CTT-3' and reverse, 5'-GGT ACA TCT CCC TGT TGA CG-3'; caspase-3 forward, 5'-GGA CCT GTG GAC CTG AAA-3'; and reverse, 5'-GGG TGC GGT AGA GTA AGC-3'; caspase-9 forward, 5'-GCC TCA TCA TCA ACA ACG-3'; and reverse, 5'-CTG GTA TGG GAC AGC ATC T-3'; and $\beta$-actin forward, 5'-GAG AGG GAA ATC GTG CGT GAC-3'; and reverse, 5'-CAT CTG CTG GAA GGT GGA CA-3'. The DNA bands were analyzed by gel electrophoresis (1.5\% agarose) and were examined using a 

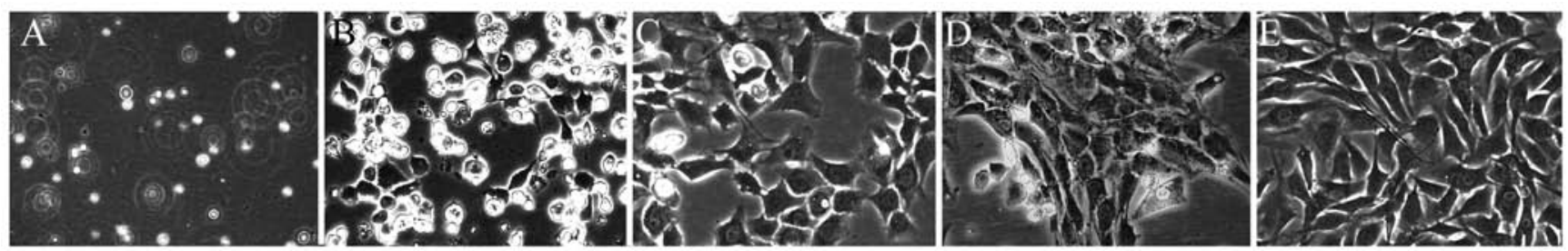

Figure 1. Morphology and identification of chondrocytes (magnification, x200). (A) Newly-isolated chondrocytes. Primary cells cultured (B) for $24 \mathrm{~h}$, (C) 4 days and (D) 8 days. (E) P2 chondrocytes cultured for 4 days.

A

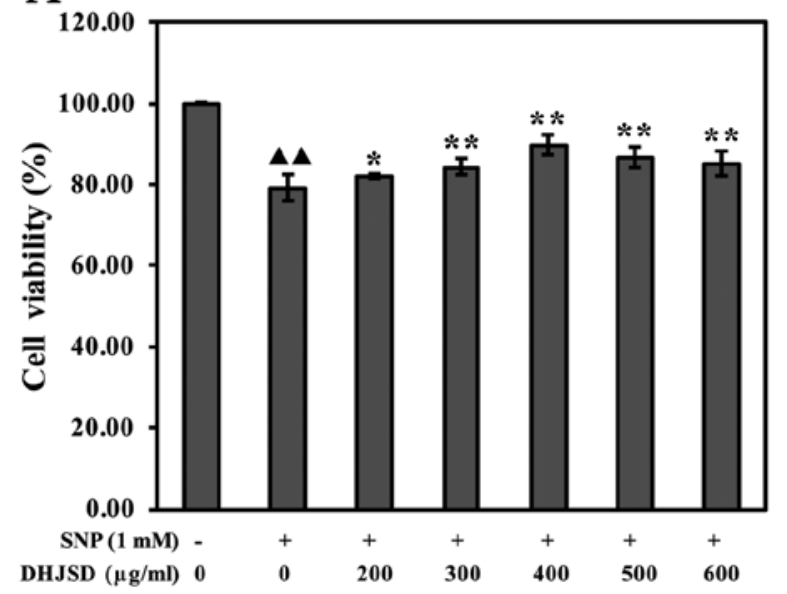

B

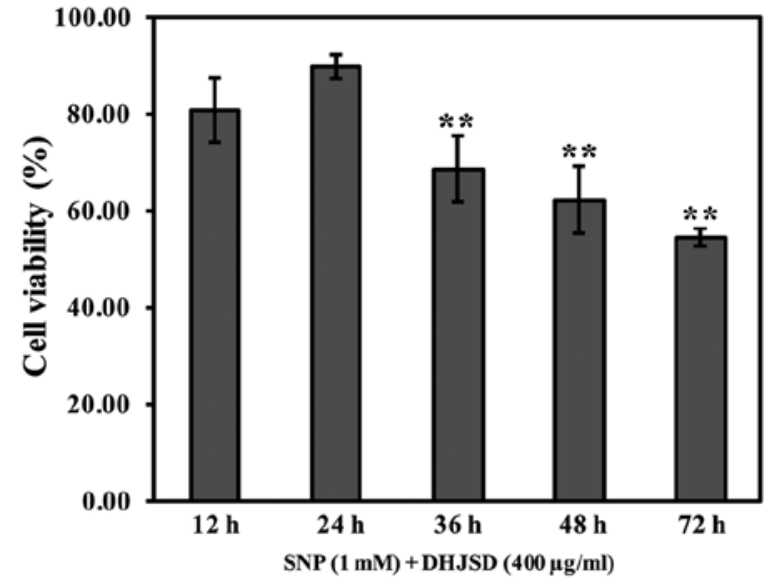

Figure 2. Duhuo Jisheng decoction (DHJSD) enhanced sodium nitroprussiate (SNP)-induced chondrocyte viability. (A) Chondrocytes treated with or without $1 \mathrm{mM}$ SNP and various DHJSD concentrations for $24 \mathrm{~h} .{ }^{\wedge}{ }^{\wedge} \mathrm{P}<0.01$ compared to untreated cells (without SNP and DHJSD); ${ }^{* *} \mathrm{P}<0.01,{ }^{*} \mathrm{P}<0.05$ compared to SNP-induced chondrocytes. (B) SNP-induced chondrocytes treated with $400 \mu \mathrm{g} / \mathrm{ml} \mathrm{DHJSD}$ for various time. ${ }^{* *} \mathrm{P}<0.01$ compared to $24 \mathrm{~h}$.

Gel Documentation System (Model Gel Doc 2000; Bio-Rad, Hercules, CA, USA) and $\beta$-actin was used as a reference gene.

Western blot analysis. Total proteins were extracted from the treated cells using the radioimmunoprecipitation assay lysis buffer (Beyotime Biotechnology, Shanghai, China) containing $1 \mathrm{mM}$ phenylmethanesulfony fluoride (Beyotime Biotechnology) and quantified using the bicinchoninic acid assay. Proteins were separated by electrophoresis on $12 \%$ SDS-polyacrylamide gels and transferred onto polyvinylidene fluoride membranes. Subsequent to blocking with 5\% skimmed milk, the membranes were incubated with primary antibodies rabbit anti-Bcl-2, rabbit anti-Bax, rabbit anti-caspase-3 and rabbit anti-caspase-9 (Cell Signaling Technology, Inc., Beverly, MA, USA) or rabbit anti- $\beta$-actin (Santa Cruz Biotechnology, Inc., Santa Cruz, CA, USA) overnight at $4^{\circ} \mathrm{C}$, and incubated with secondary horseradish peroxidase-conjugated immunoglobulin $\mathrm{G}$ antibody (Zhongshan Golden Bridge Biotechnology, Co., Ltd., Beijing, China). Electrochemiluminescence was used to make the signal visible, and images were captured using a Bio-Rad Chemi Doc $\mathrm{XRS}^{+}$(Bio-Rad), prior to normalizing to that of $\beta$-actin.

Statistical analysis. Data were expressed as the mean \pm standard deviation from at least three independent experiments. Statistical analysis was performed by one-way analysis of variance or Student's t-test using SPSS 19.0 (IBM, Corp., Armonk, NY, USA). $\mathrm{P}<0.05$ was considered to indicate a statistically significant difference.

\section{Results}

Morphology and identification of chondrocytes. The chondrocyte morphology was as described previously (Fig. 1) $(16,17)$. Newly-isolated chondrocytes were known as passage 0 (P0). P2 chondrocytes are rich in extracellular matrix and optimum with good cell vitality and morphology.

DHJSD enhances SNP-induced chondrocyte viability. To investigate the effects of DHJSD on cell viability, SNP-induced chondrocytes were treated with various concentrations of DHJSD for different times, and subsequently subjected to the MTT assay. The results showed that the viability of SNP-induced chondrocytes was significantly lower than that of the untreated cells $(\mathrm{P}<0.01)$ and the viability of SNP-induced chondrocytes treated by DHJSD was higher compared to the SNP-induced chondrocytes $(\mathrm{P}<0.05)$ (Fig. 2A). The viability of SNP-induced chondrocytes treated by $400 \mu \mathrm{g} / \mathrm{ml}$ DHJSD for $24 \mathrm{~h}$ was higher compared to 36,48 and $72 \mathrm{~h}$, respectively $(\mathrm{P}<0.01)$ (Fig. $2 \mathrm{~B})$, indicating that DHJSD enhanced SNP-induced chondrocyte viability in a dose- and time-dependent manner. Therefore, $1 \mathrm{mM} \mathrm{SNP}$ and various concentrations $(300,400$ and $500 \mu \mathrm{g} / \mathrm{ml})$ of DHJSD for $24 \mathrm{~h}$ were used in the following experiments.

Effects of DHJSD on the morphology changes of SNP-induced chondrocytes. To observe the morphology changes of SNP-induced chondrocytes treated by DHJSD, the morphology was observed by a phase-contrast microscope (Figs. 3 and 4). 


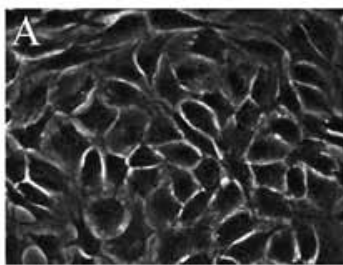

SNP $(1 \mathrm{mM})$

$\operatorname{DHJSD}(\mu \mathrm{g} / \mathrm{ml}) \quad 0$

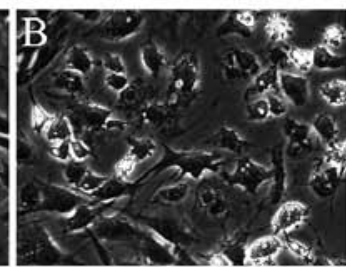

$+$

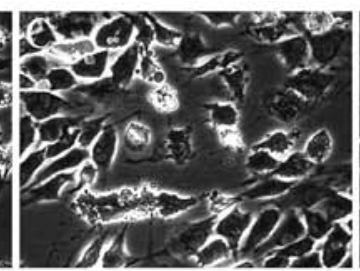

$+$

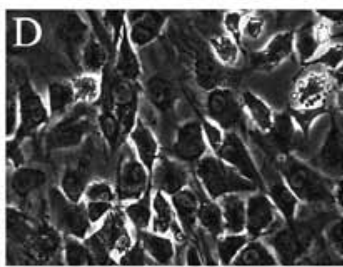

$+$

400

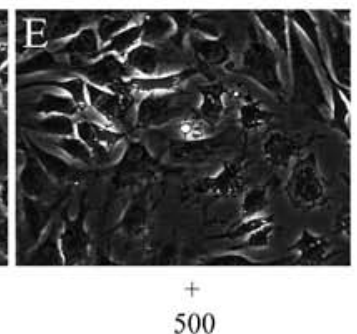

500

Figure 3. Effect of Duhuo Jisheng decoction (DHJSD) on the morphology changes of sodium nitroprussiate (SNP)-induced chondrocytes (magnification, x200). (A) Untreated cells. (B) SNP-induced chondrocytes. (C) SNP-induced chondrocytes treated by 300, (D) 400 or (E) $500 \mu \mathrm{g} / \mathrm{ml}$ DHJSD.

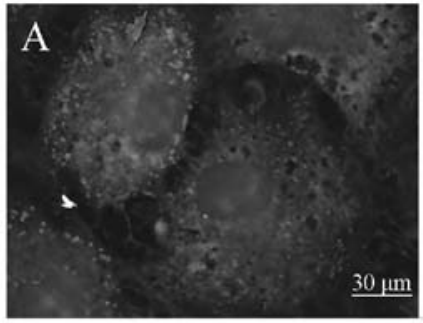

$\mathrm{SNP}(1 \mathrm{mM})$ $\operatorname{DHJSD}(\mu \mathrm{g} / \mathrm{ml}) \quad 0$

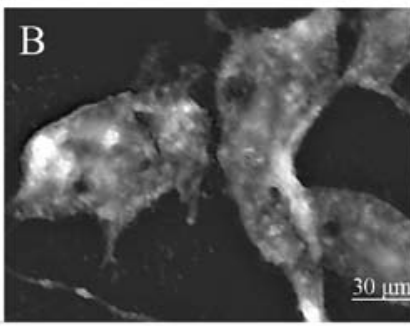

$+$

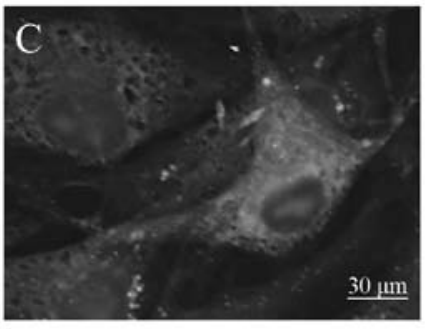

$+$

Figure 4. Scanning electron micrograph of sodium nitroprussiate (SNP)-induced chondrocytes. (A) Untreated cells. (B) SNP-induced chondrocyte. (C) SNP-induced chondrocyte treated by $400 \mu \mathrm{g} / \mathrm{ml}$ Duhuo Jisheng decoction (DHJSD).

Untreated cells showed that the morphology of chondrocytes in culture was indicative of the healthy status of the cells, whereas SNP-induced chondrocytes exhibited apoptotic characteristic cells that became rounded, bright and contracted, and detached from each other or floated in the medium, compared to the SNP-induced chondrocytes treated by DHJSD.

DHJSD inhibits the apoptosis of SNP-induced chondrocytes. The chondrocytes were identified by collagen type II immunohistochemistry. The cytoplasm of positive chondrocytes was stained brown, whereas the negative control failed to stain in the cytoplasm (Fig. 5A and B). To examine whether DHJSD enhanced SNP-induced chondrocyte viability by suppressing apoptosis, the apoptotic cells were determined by DAPI staining. Apoptotic cells exhibited typical changes, including reduction of cellular volume, bright blue staining and condensed or fragmented nuclei. This phenomenon was more clear in SNP-induced chondrocytes than that of the SNP-induced chondrocytes treated by DHJSD (Fig. 5C). To further investigate the effect of DHJSD on the apoptosis of SNP-induced chondrocyte, chondrocyte apoptosis was measured by Annexin V/PI staining. Apoptosis is shown in Fig. 5D; upper right quadrant, late apoptotic cells; upper left quadrant, dead cells; lower left quadrant, normal cells; and lower right quadrant, early apoptotic cells. The percentage of apoptotic cells (including the early and late apoptotic cells, and dead cells) in SNP-induced chondrocytes treated by DHJSD were significantly lower than that of the SNP-induced chondrocytes $(\mathrm{P}<0.01)$ (Fig. 5F), which implied that DHJSD inhibited SNP-induced chondrocyte apoptosis.

DHJSD decreases the mitochondrial membrane potential $(\Delta \Psi m)$ of SNP-induced chondrocytes. JC-1 converts to the monomeric form within the cytoplasm, which is caused by the loss of mitochondrial membrane potential. To investigate the effect of DHJSD on the loss of $\Delta \Psi \mathrm{m}$, a typical early event of apoptosis, JC-1 staining analysis was used to measure the changes of $\Delta \Psi \mathrm{m}$. As shown in Fig. 5E, the cells with a loss of $\Delta \Psi \mathrm{m}$ were revealed by the decrease of red fluorescence (R2). The percentage of live cells (R2) in SNP-induced chondrocytes treated by DHJSD had a greater number of cells compared to the SNP-induced chondrocytes $(\mathrm{P}<0.01)$ (Fig. 5G), suggesting that DHJSD decreased the loss of $\Delta \Psi \mathrm{m}$ in SNP-induced chondrocytes.

DHJSD increases Bcl-2 and decreases Bax, caspase-9 and caspase-3 expression. The mitochondrial-dependent signaling pathway is controlled by the Bcl-2 family proteins, such as anti-apoptotic member Bcl-2 and pro-apoptotic member Bax. The anti-apoptotic protein $\mathrm{Bcl}-2$ suppresses cell apoptosis, and the expression of Bax results in the release of numerous apoptogenic proteins from the mitochondria triggering the activation of caspase- 9 and caspase-3, and eventually inducing apoptosis. To further study the mechanism of DHJSD on SNP-induced chondrocyte apoptosis, the expressions of Bax, Bcl-2, caspase- 9 and caspase- 3 were detected by RT-PCR and western blot analysis, respectively. The RT-PCR assay showed that in the SNP-induced chondrocytes treated by DHJSD, the Bcl-2 expression was extremely increased, whereas the Bax, caspase-9 and caspase-3 expressions were decreased, compared to that of the SNP-induced chondrocytes $(\mathrm{P}<0.01)$ (Fig. 6). The protein levels of Bax, Bcl-2, caspase-3 and caspase-9 was similar to their respective mRNA expression $(\mathrm{P}<0.01)$ (Fig. 7). Taken together, the results indicated that DHJSD inhibits SNP-induced chondrocyte apoptosis via the mitochondrial-dependent signaling pathway. 


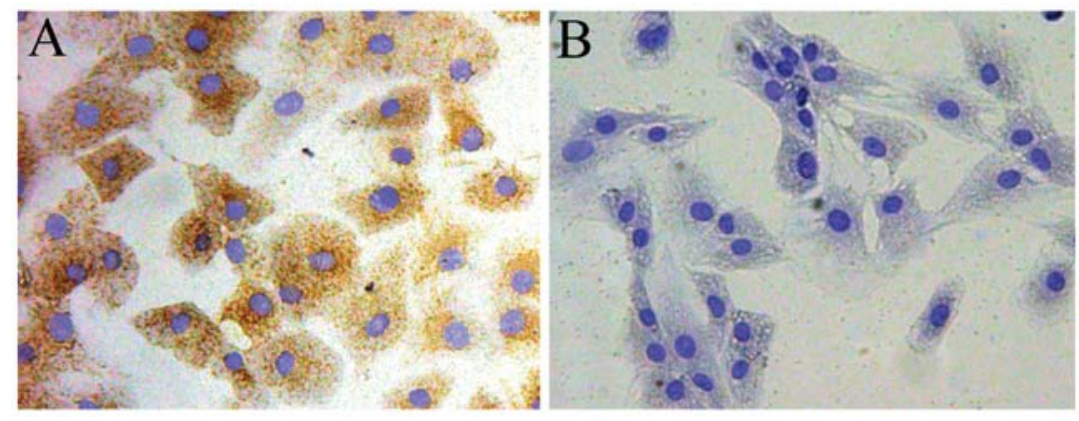

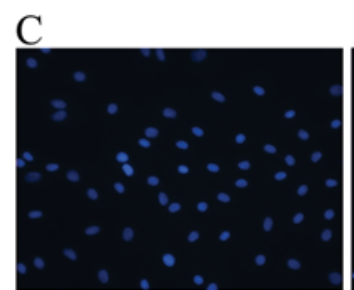

SNP $(1 \mathrm{mM})$ $\operatorname{DHJSD}(\mu \mathrm{g} / \mathrm{ml}) \quad 0$

$\mathrm{D}$

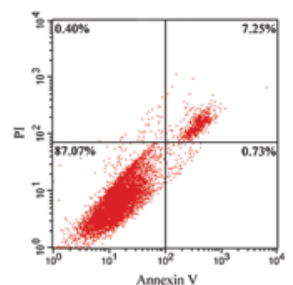

SNP $(1 \mathrm{mM})$ $\operatorname{DHJSD}(\mu \mathrm{g} / \mathrm{ml}) \quad 0$

$\mathrm{E}$

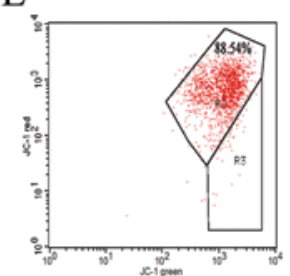

SNP (1 mM) $\operatorname{DHJSD}(\mu \mathrm{g} / \mathrm{ml}) \quad 0$

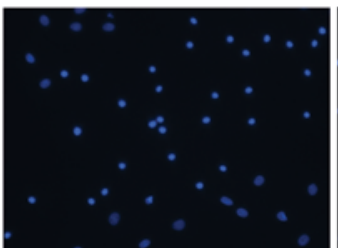

$+$

0
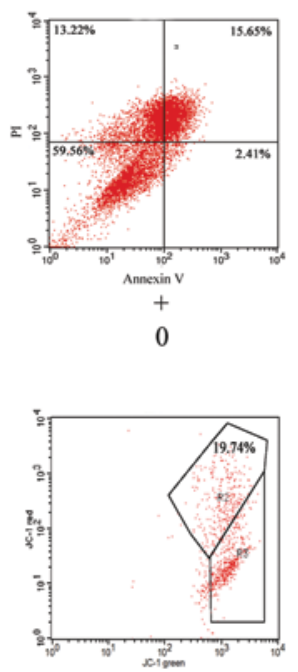

0

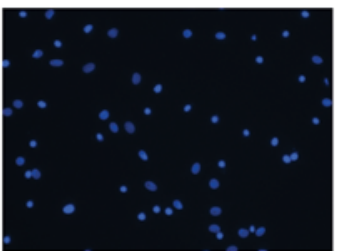

$+$

300

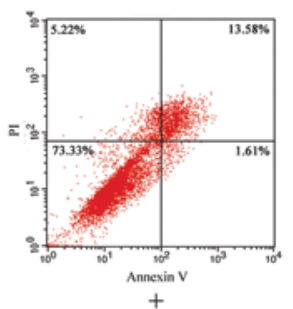

300

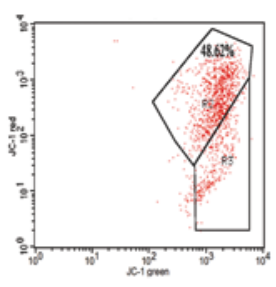

300

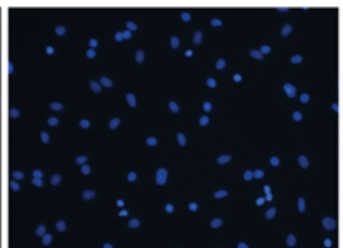

$+$

400

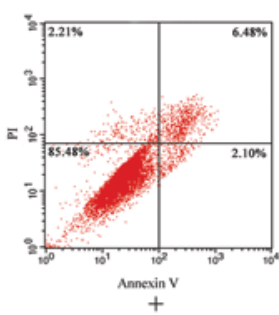

400

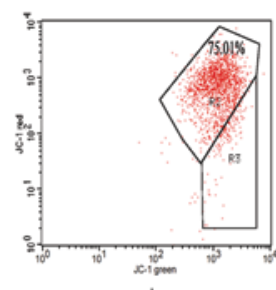

400

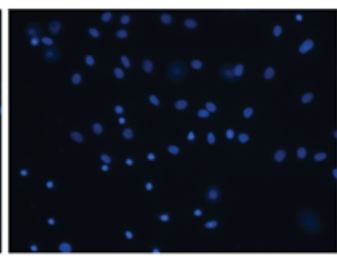

$+$

500

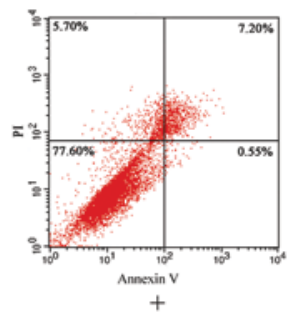

500

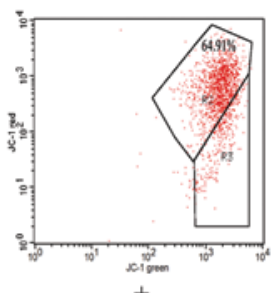

500
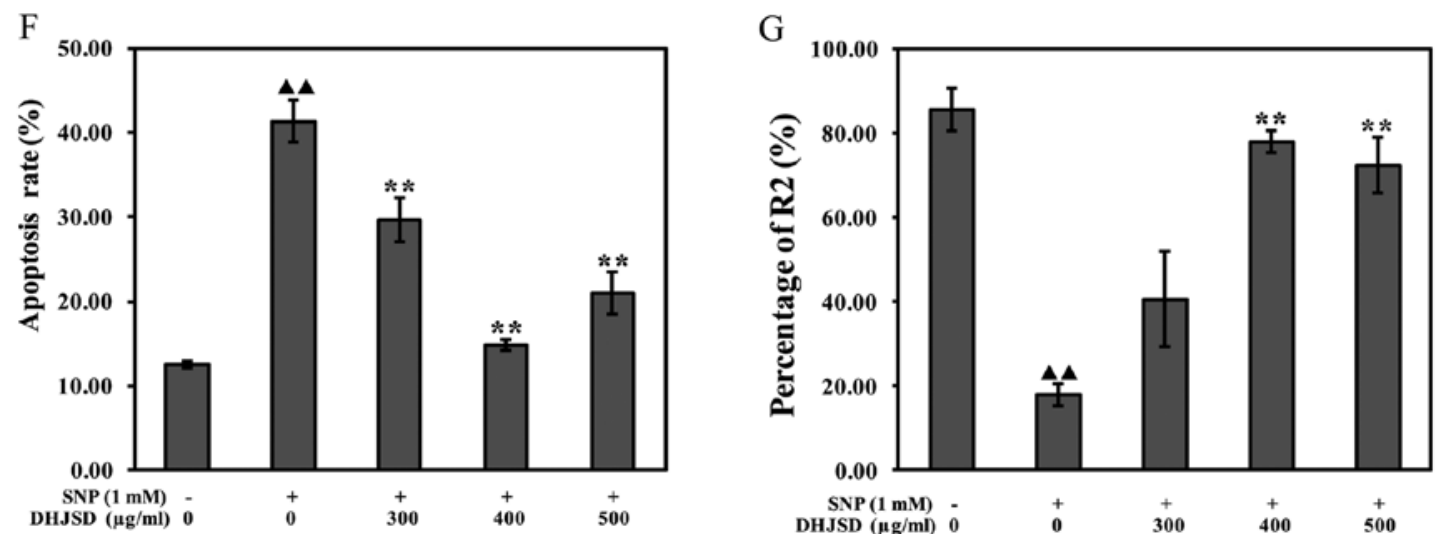

Figure 5. Duhuo Jisheng decoction (DHJSD) inhibited the apoptosis and the mitochondrial membrane potential $(\Delta \Psi \mathrm{m})$ of sodium nitroprussiate (SNP)-induced chondrocytes. (A) P2 chondrocytes cultured for 4 days, identified by collagen type II immunohistochemistry. (B) P2 chondrocytes cultured for 4 days as the negative control identified by collagen type II immunohistochemistry. (C) Chondrocyte apoptosis morphology change was examined by 4',6-diamidino-2-phenylindole (DAPI) staining. (D) Chondrocyte apoptosis rate was assessed by Annexin V/propidium iodide (PI) staining. (E) The changes of $\Delta \Psi \mathrm{m}$ in chondrocytes treated with or without SNP and various DHJSD concentrations were revealed by JC-1 staining. (F) Percentage of apoptosis in chondrocytes treated with or without SNP and various DHJSD concentrations. (G) Percentage of live cells (R2) in chondrocytes treated with or without SNP and various DHJSD concentrations. ${ }^{\wedge} \mathrm{P}<0.01$ compared to untreated cells; ${ }^{* *} \mathrm{P}<0.01$, compared to SNP-induced chondrocytes. 

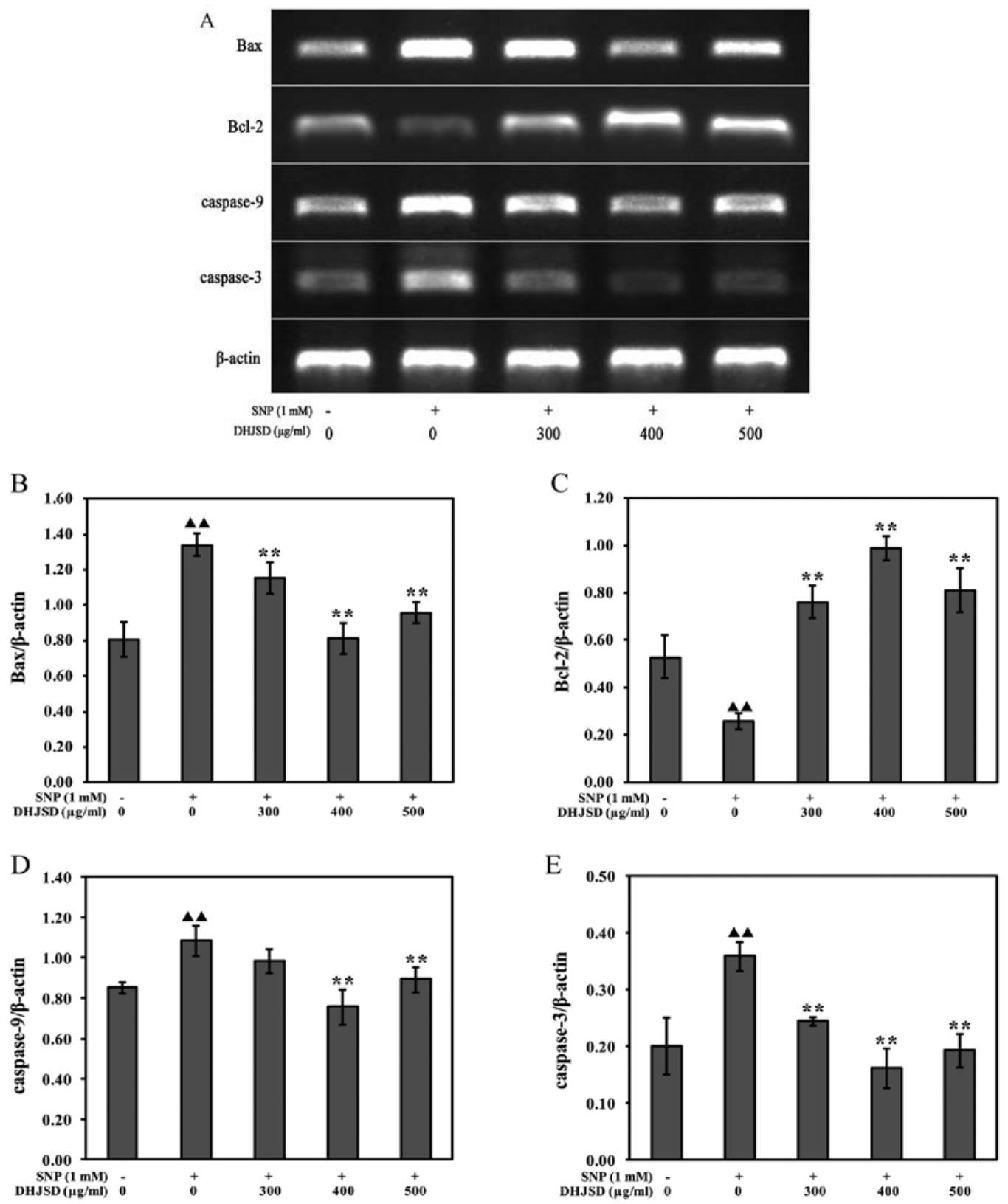

Figure 6. Duhuo Jisheng decoction (DHJSD) increased the mRNA expression of $B c l-2$, and decreased the mRNA expression of Bax, caspase-9 and caspase-3. (A) mRNA expression levels of Bax, Bcl-2, caspase-9 and caspase-3; $\beta$-actin was used as the internal control. (B) mRNA expression levels of Bax, (C) Bcl-2, (D) caspase-9 and (E) caspase-3. ${ }^{\wedge} \mathrm{P}<0.01$ compared to untreated cells; ${ }^{* *} \mathrm{P}<0.01$, compared to SNP-induced chondrocytes.

\section{Discussion}

Chondrocyte apoptosis leads to cartilage degeneration $(18,19)$, thus, inhibiting chondrocyte apoptosis may be an efficient method for the treatment of OA. The present results showed that DHJSD inhibits SNP-induced chondrocyte apoptosis by upregulating the protein and mRNA expression of Bcl-2, whereas downregulating the protein and mRNA expression of Bax, caspase- 9 and caspase-3 indicates that DHJSD may be a potential agent for treating OA.

OA is a chronic disease and thus far there is no radical therapy available (20), and only several drugs, such as non-steroidal anti-inflammatory drugs and cyclooxygenase- 2 inhibitors, are used to treat OA, but they cause serious side-effects. Therefore, novel therapeutic methods require development for reducing the disease burden of OA. Chinese medical herbs, which are safe and cheap, have been used to alleviate symptoms and delay the pathological progress of OA for thousands of years. DHJSD has been proved effective in OA treatment by clinical practice (21). However, the underlying mechanisms for the management of OA are not fully understood. In addition, NO has been shown to be a key inducer of chondrocyte apoptosis, a central pathogenic feature of OA $(22,23)$. SNP, an NO donor compound, induces chondrocyte apoptosis via the mitochondrial-dependent signaling (24). To explore the effects of DHJSD on SNP-induced chondrocyte, SNP-induced chondrocyte apoptosis was used as the mitochondrial-dependent apoptotic model.

The MTT assay is based on the reduction of tetrazolium salt by mitochondrial succinate dehydrogenases in viable cells yielding purple formazan crystals, whereas dead cells do 

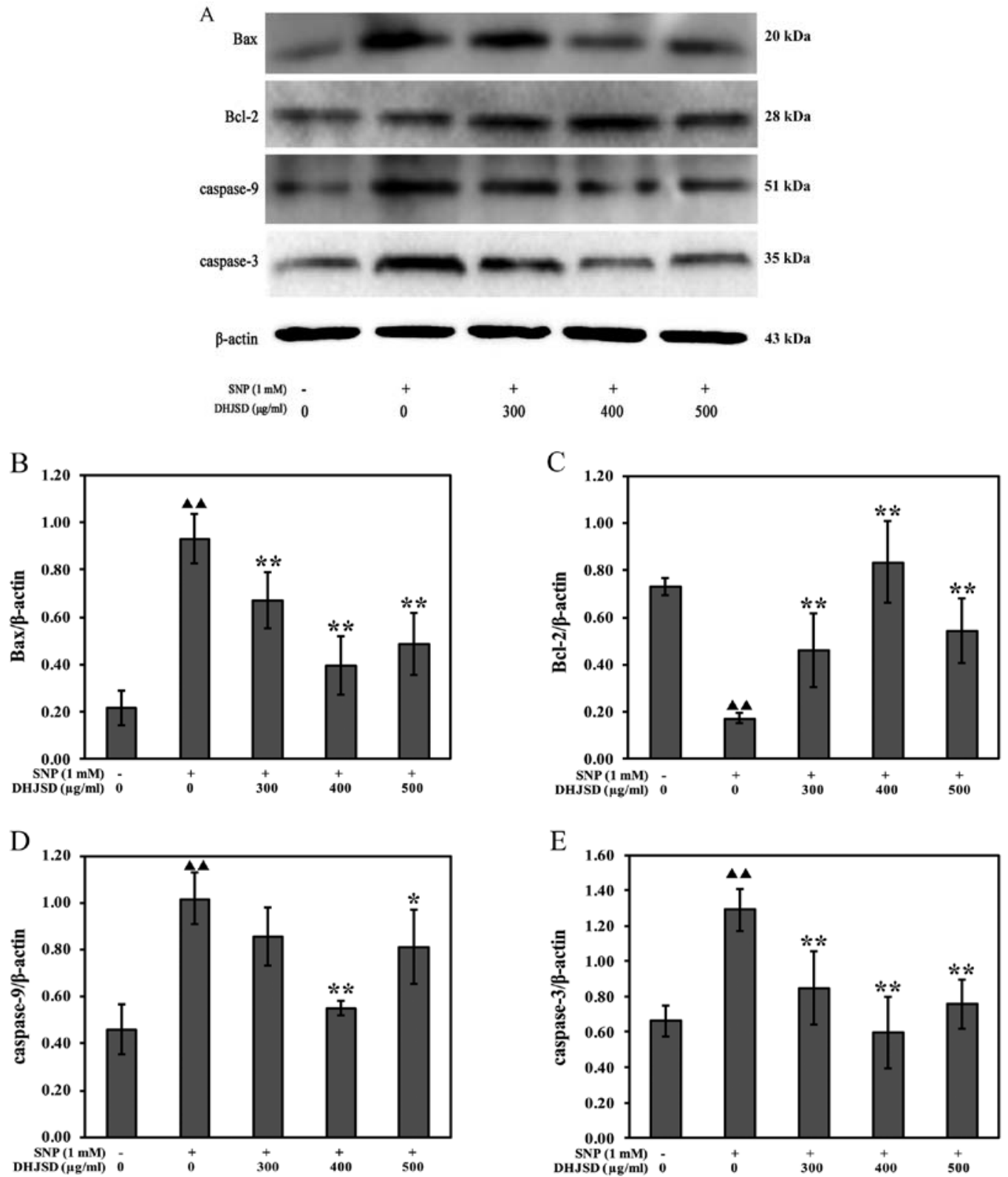

Figure 7. Duhuo Jisheng decoction (DHJSD) increased the protein expression of B-cell lymphoma 2 (Bcl-2), and decreased the protein expression of Bax, caspase-9 and caspase-3. (A) Protein levels of Bax, Bcl-2, caspase-9 and caspase-3; $\beta$-actin was used as the internal control. (B) Protein levels of Bax, (C) Bcl-2, (D) caspase-9 and (E) caspase-3. ${ }^{\wedge} \mathrm{P}<0.01$ compared to untreated cells, ${ }^{* *} \mathrm{P}<0.01,{ }^{*} \mathrm{P}<0.05$ compared to sodium nitroprussiate (SNP)-induced chondrocytes.

not yield crystals. Therefore, the monitoring of alterations in mitochondrial activity can be detected by the MTT assay. The concentration of MTT formazan is directly proportional to the number of viable cells (25). A screening method was used in the present study to measure SNP-induced chondrocyte viability by MTT. According to the results from the MTT assay, DHJSD enhanced SNP-induced chondrocyte viability in a dose- and time-dependent manner. To study this further, Annexin V/PI and DAPI staining were used to explore the effect of DHJSD on apoptosis of SNP-induced chondrocyte. Annexin V is a 35-36 $\mathrm{kDa} \mathrm{Ca}{ }^{2+}$-dependent phospholipid-binding protein that has a high affinity for membrane phospholipid phosphatidylserine (PS), which is translocated from the inner to the outer leaflet of the plasma membrane in apoptotic cells. This allowed the number of apoptosis-positive cells to be expressed as a percentage of total cells. Therefore, flow cytometry with Annexin V/PI staining was used to further confirm the percentage of apoptosis, which decreased in a dose-dependent manner. The loss of $\Delta \Psi \mathrm{m}$, a characteristic of apoptosis, is an early event preceding phosphatidylserine externalization and coincides with caspase activation. JC-1 is sensitive to $\Delta \Psi \mathrm{m}$ and the changes in the ratio between red and green fluorescence provide information regarding the mitochondrial membrane potential by forming aggregates that have a fluorescence emission spectra peak at $590 \mathrm{~nm}$ (red) at high $\Delta \Psi \mathrm{m}$, and becoming a monomer again with a fluorescence emission peak at $530 \mathrm{~nm}$ (green) at low $\Delta \Psi \mathrm{m}$. Therefore, JC-1 staining assay was used to evaluate mitochondrial membrane potential and data showed that DHJSD reduced the collapse of $\Delta \Psi \mathrm{m}$.

The mitochondrial-dependent signaling pathway is the center of apoptosis control, and is relient on mitochondrial outer membrane permeabilisation (MOMP), which is regulated by the 
pro-apoptotic Bcl-2 family proteins, such as Bax and Bcl-2 (26). Bax, a direct pro-apoptotic effector of MOMP, translocates from the cytosol to the outer mitochondrial membrane (OMM) and oligomerizes, wherein it contributes to the formation of pores to release cytochrome $c$ that stimulates the activation of caspase-9 and caspase- $3(27,28)$. Caspase activation is usually regulated by the Bcl-2 family (29). Bcl-2, one of the anti-apoptotic proteins that are located in the OMM, is a mitochondrial membrane-associated protein, and exerts its anti-apoptotic effect by inhibiting Bax expression from mitochondria and reducing the release of cytochrome $c$, as well as subsequently inhibiting the activation of caspase-3 $(30,31)$. Activation of upstream caspases- 9 leads to the proteolytic activation of downstream or effector caspases-3, a marker of apoptosis. Cytosolic caspase-3 is cleaved and exerts final execution of apoptosis through degeneration of vital proteins, resulting in cell destruction by apoptosis via the cleavage of structural and regulatory proteins. Whether DHJSD inhibits mitochondrial-dependent signaling pathway to regulate chondrocytes apoptosis was investigated. The mRNA and protein expression of Bax, Bcl-2, caspase- 9 and caspase- 3 were detected by RT-PCR and western blot analysis, respectively. The results showed that DHJSD upregulates the mRNA and protein expression of $\mathrm{Bcl}-2$, and downregulates the mRNA and protein expression of Bax, caspase-9 and caspase-3.

In conclusion, DHJSD inhibits SNP-induced chondrocyte apoptosis by the mitochondrial-dependent signaling pathway, indicating that DHJSD may be a potential novel therapeutic agent for the treatment of OA. These results may in part explain the mechanisms by which DHJSD exerts its beneficial effects in OA, and have certain limits. Using a mitochondrion-dependent apoptotic pathway signal inhibitor and exploring in vivo are required in future studies.

\section{Acknowledgements}

The present study was supported by the Key Project of Fujian Provincial Department of Science and Technology (grant no. 2012Y0046) and the Young Talent Scientific Research Project of Fujian Province Universities (grant no. JA12165).

\section{References}

1. Thomas CM, Fuller CJ, Whittles CE and Sharif M: Chondrocyte death by apoptosis is associated with the initiation and severity of articular cartilage degradation. Int J Rheum Dis 14: 191-198, 2011.

2. Usichenko TI, Edinger H, Witstruck T, et al: Millimetre wave therapy for pain relief after total knee arthroplasty: a randomised controlled trial. Eur J Pain 12: 617-623, 2008.

3. Adams JM and Cory S: The Bcl-2 apoptotic switch in cancer development and therapy. Oncogene 26: 1324-1337, 2007.

4. Knudson CM and Brown NM: Mitochondria potential, bax 'activation', and programmed cell death. Methods Mol Biol 414: 95-108, 2008.

5. Gupta S, Kass GE, Szegezdi E and Joseph B: The mitochondrial death pathway: a promising therapeutic target in diseases. J Cell Mol Med 13: 1004-1033, 2009.

6. Rego I, Fernández-Moreno M and Fernández-López C, et al: Role of European mitochondrial DNA haplogroups in the prevalence of hip osteoarthritis in Galicia, Northern Spain. Ann Rheum Dis 69: 210-213, 2010.

7. Edmonds S: Therapeutic targets for osteoarthritis. Maturitas 63 : 191-194, 2009

8. Altman R and Barkin RL: Topical therapy for osteoarthritis: clinical and pharmacologic perspectives. Postgrad Med 121: 139-147, 2009.
9. Hua B, Abbas E, Hayes A, Ryan P, Nelson L and O'Brien K: Reliability of Chinese medicine diagnostic variables in the examination of patients with osteoarthritis of the knee. J Altern Complement Med 18: 1028-1037, 2012.

10. Wang X, Wei S, Liu T, et al: Effectiveness, medication patterns, and adverse events of traditional Chinese herbal patches for osteoarthritis: a systematic review. Evid Based Complement Alternat Med 2014: 1-17, 2014.

11. Lai J, Chen H, Chen C, Lin J, Hwang J and Wang J: Duhuo Jisheng Tang for treating osteoarthritis of the knee: a prospective clinical observation. Chin Med 2: 1-8, 2007.

12. Zheng CS, Xu XJ, Ye HZ, et al: Computational approaches for exploring the potential synergy and polypharmacology of Duhuo Jisheng Decoction in the therapy of osteoarthritis. Exp Ther Med 7: 1163-1168, 2013

13. Chinese Pharmacopeia Commission: Pharmacopoeia of the People's Republic of China. Chinese Medical Science and Technology Press, Beijing, China, 2010.

14. Li X, Du M, Liu X, et al: Millimeter wave treatment inhibits NO-induced apoptosis of chondrocytes through the p38MAPK pathway. Int J Mol Med 25: 393-399, 2010.

15. Li X, Du M, Liu X, et al: Millimeter wave treatment promotes chondrocyte proliferation by upregulating the expression of cyclin-dependent kinase 2 and cyclin A. Int J Mol Med 26: 77-84, 2010.

16. Yu F, Li X, Cai L, et al: Achyranthes bidentata polysaccharides induce chondrocyte proliferation via the promotion of the G1/S cell cycle transition. Mol Med Rep 7: 935-940, 2013.

17. Li H, Li X, Liu G, et al: Bauhinia championi (Benth.) Benth. polysaccharides upregulate $\mathrm{Wnt} / \beta$-catenin signaling in chondrocytes. Int J Mol Med 32: 1329-1336, 2013.

18. Chan BY, Fuller ES, Russell AK, et al: Increased chondrocyte sclerostin may protect against cartilage degradation in osteoarthritis. Osteoarthr Cartilage 19: 874-885, 2011.

19. Huang JG, Xia C, Zheng XP, et al: $17 \beta$-Estradiol promotes cell proliferation in rat osteoarthritis model chondrocytes via PI3K/Akt pathway. Cell Mol Biol Lett 16: 564-575, 2011.

20. Bijlsma JW, Berenbaum F and Lafeber FP: Osteoarthritis: an update with relevance for clinical practice. Lancet 377: 2115-2126, 2011.

21. Hsieh S, Lai J, Chen P, Chen C, Chen H and Wang J: Is Duhuo Jisheng Tang containing Xixin safe? A four-week safety study. Chin Med 5: 1-6, 2010.

22. Wu GJ, Chen TG, Chang HC, Chiu WT, Chang CC and Chen RM: Nitric oxide from both exogenous and endogenous sources activates mitochondria-dependent events and induces insults to human chondrocytes. J Cell Biochem 101: 1520-1531, 2007.

23. Maneiro E, López-Armada MJ, de Andres MC, et al: Effect of nitric oxide on mitochondrial respiratory activity of human articular chondrocytes. Ann Rheum Dis 64: 388-395, 2005.

24. Tonomura H, Takahashi KA, Mazda O, et al: Glutamine protects articular chondrocytes from heat stress and NO-induced apoptosis with HSP70 expression. Osteoarthr Cartilage 14: 545-553, 2006.

25. Etxeberria A, Mendarte S and Larregla S: Determination of viability of Phytophthora capsici oospores with the tetrazolium bromide staining test versus a plasmolysis method. Rev Iberoam Micol 28: 43-49, 2011.

26. Renault TT, Teijido O, Antonsson B, Dejean LM and Manon S: Regulation of Bax mitochondrial localization by Bcl-2 and Bcl-x (L): keep your friends close but your enemies closer. Int J Biochem Cell Biol 45: 64-67, 2013.

27. Westphal D, Dewson G, Czabotar PE and Kluck RM: Molecular biology of Bax and Bak activation and action. Biochim Biophys Acta 1813: 521-531, 2011.

28. Renault TT and Manon S: Bax: Addressed to kill. Biochimie 93: 1379-1391, 2011.

29. Chai WS, Zu XM, Li SH, Fan JX and Chen BY: Role of Bcl-2 family members in caspase-3/9-dependent apoptosis during Pseudomonas aeruginosa infection in U937 cells. Apoptosis 13: 833-843, 2008.

30. Lu H, Chie Y, Yang M, et al: Apigenin induces caspase-dependent apoptosis in human lung cancer A549 cells through Bax-and Bcl-2-triggered mitochondrial pathway. Int J Oncol 36: $1477-1484,2010$.

31. Autret A and Martin SJ: Emerging role for members of the Bcl-2 family in mitochondrial morphogenesis. Mol Cell 36: 355-363, 2009. 